\title{
Particle Swarm Optimization Based LQ-Servo Controller for Congestion Avoidance
}

\author{
Sana S. Sabry ${ }^{1}$, Thaker M. Nayl ${ }^{2}$ \\ ${ }^{1}$ college of engineering, University of information technology and communications, Baghdad, Iraq \\ ${ }^{2}$ college of engineering, University of information technology and communications, Baghdad, Iraq \\ sana.sabah@uoitc.edu.iq, thaker.nayl@uoitc.edu.iq
}

\begin{abstract}
The network congestion is an essential problem that leads to packets losing and performance degradation. Thus, preventing congestion in the network is very important to enhance and improve the quality of service. Active queue management (AQM) is the solution to control congestion in TCP network middle nodes to improve theire performance. We design a linear quadratic (LQ)-servo controller as an AQM applied to TCP network to control congestion and attempt to achieve high quality of service under dynamic network environments. The LQ-servo controller is proposed to provide queue length stabilization with a small delay and faster settling time. The designed controller parameters are tuned by using the particle swarm optimization (PSO) method. The PSO algorithm was fundamentally applied to find the optimal controller parameters $Q$ and $R$, such that a good output response could be obtained. The PI controller is examined for comparison reasons. The MATLAB simulation result shows that the controller is more effective than the PI in reaching zero steadystate error with better congestion avoidance under the dynamic network environment. Moreover, the proposed controller achieves a smaller delay and faster settling time.
\end{abstract}

Index Terms - AQM, Network Congestion control, LQ-servo, Particle Swarm Optimization.

\section{INTRODUCTION}

With the fast evolution of communication networks and the internet, the large amount of generated data requires a reliable and effective data transmission to avoid network congestion. Thus, preventing congestion in the network is very important to enhance and improve the quality of service.

Transmission control protocol is one of the most widespread transport protocols which provides an end to end congestion control mechanism [1]. In this protocol, the average congestion window size increases when the packets are delivered to the receiver. Conversely, the average congestion window size decreases during an unsuccessful data transition. However, this mechanism has significant disadvantages such as poor utilization for the network resources and global stream synchronization [2]. To avoid these disadvantages, AQM has been proposed at gateways to improve their performance. Several types of AQM in recent years have been proposed to control congestion in TCP networks. in [3] random early detection (RED) was proposed which randomly dropped the packet at specific probability before the queue buffer overflows. Then Misra in [4] obtained numerically transient behaviour of AQM/TCP routers. This modelling has a great role in understanding and analyzing different network congestion avoidance schemes. In [5], the linearization was used by Hollot et al. to analyze the previously obtained non-linear model for the AQM /TCP routers. A PI controller was proposed in [6] based on the linearized model and the control theory, the controller displayed better properties compared to the RED controller. In [7], a linear quadratic (LQ)-servo controller was 
proposed and the controller parameter was chosen by trial and error. In [8], an adaptive AQM based neural network was developed and the proposed algorithm showed a good performance.

All the AQM proposed above do not control the congestion of a dynamic network environment, where the parameters of the network change continuously.

In this paper, an improved AQM based on PSO- LQ controller is designed to control congestion and attempt to achieve high quality of service under the various network environments.

The paper contributions are as follows:

- An LQ-servo controller is proposed to provide queue length stabilization with a small delay and faster settling time.

- The LQ-servo controller is compared with the PI controller.

- A PSO algorithm is applied to tune the controller parameter.

- The steadiness of the controller is examined under different network environments.

This paper is structured as follows: section II describes the linearized AQM model, while the linear quadratic controller is discussed in section III. Afterwards, section IV gives an overview about the practical swarm optimization method. Section V shows the MATLAB simulation results. Finally, section VI discusses the findings and conclusions of this research.

\section{TCP/ AQM SYSTEM DYNAMICS}

\section{A. TCP Model}

The dynamic behaviour of the TCP model has been developed by [4] using stochastic differential equation analysis and fluid flow, and it is expressed by:

$$
\begin{aligned}
& \dot{W}(t)=\frac{1}{r(t)}-\frac{W(t) W(t-r(t))}{2 r(t-r(t))} p(t-r(t)) \\
& \dot{q}(t)=\frac{W(t)}{r(t)} N(t)-C
\end{aligned}
$$

Where $W$ is the congestion window size (packet).

$q$ is the queue size (packet).

$r: q / C+T_{p}$ is the Round-trip time (second).

$C$ : is the single link capacity (packets/second).

$N$ : are TCP connections.

$P$ : is the packet dropping probability.

$t$ : is the time (second)

\section{B. Linearization}

For control analysis purposes, (1) was linearized about an equilibrium point to get the linearized TCP model described in (2), see [6] for more linearization details.

$$
\begin{aligned}
& \delta \dot{W}(t)=-\frac{2 N}{r_{0}^{2} C} \delta W(t)-\frac{r_{0} C^{2}}{2 N^{2}} \delta p\left(t-r_{0}\right) \\
& \delta \dot{q}(t)=\frac{N}{r_{0}} \delta W(t)-\frac{1}{r_{0}} \delta q(t)
\end{aligned}
$$

Rewriting (2) in state space form leads to the next equation 


$$
\begin{aligned}
& x^{\bullet}(t)=A x(t)+B u\left(t-r_{0}\right) \\
& y(t)=C x(t)
\end{aligned}
$$

Where

$$
x(t)=\left[\begin{array}{c}
\delta W \\
\delta q
\end{array}\right]
$$

, $y(t)$ is the queue length and $u(t)$ is a packet dropping probability, as shown in Fig. 1.

$$
\begin{gathered}
A=\left[\begin{array}{cc}
-\frac{2 N}{r_{0}^{2}} & 0 \\
\frac{N}{r} & -\frac{1}{r}
\end{array}\right], B=\left[\begin{array}{c}
-\frac{r_{0} C^{2}}{2 N^{2}} \\
0
\end{array}\right] \\
C=\left[\begin{array}{ll}
0 & 1
\end{array}\right]
\end{gathered}
$$

The linearized AQM block diagram is shown in Fig.1.

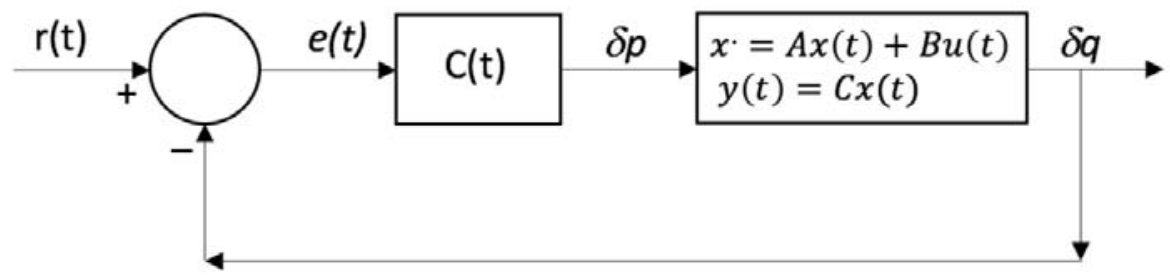

FIG. 1. LINEARIZED AQM DIAGRAM AS FEEDBACK CONTROL

Using the discrete-time theory, the continuous-time system expressed by (3) can be represented by the discrete-time system as in (5):

$$
\begin{aligned}
& \mathrm{x}_{\mathrm{d}}(\mathrm{k}+1)=\phi \mathrm{x}_{\mathrm{d}}(\mathrm{k})+\Gamma \mathrm{u}_{\mathrm{d}}(\mathrm{k}) \\
& \mathrm{y}_{\mathrm{d}}(\mathrm{k})=C \mathrm{x}_{\mathrm{d}}(\mathrm{k})
\end{aligned}
$$

Where

$$
\phi=e^{A T}, \Gamma=\left(\int_{0}^{T} e^{A T} d t\right) \text { and } \mathrm{T} \text { is the sampling period. }
$$

\section{THE CONTROLLER DESIGN}

In this section, the LQ-servo controller is presented to make the output (queue length) stabilize to the desired queue length with a minimum steady-state error $\left(e_{s s}\right)$ for network congestion avoidance. 


\section{A. Servo Structure for the TCP Model}

The main principle of the servo system is when the system model has no integrator (as the TCP model) a feedforward integrator must be inserted as shown in Fig 2 to meet the controller objective in reaching zero $e_{s s}$ in the system response [9].

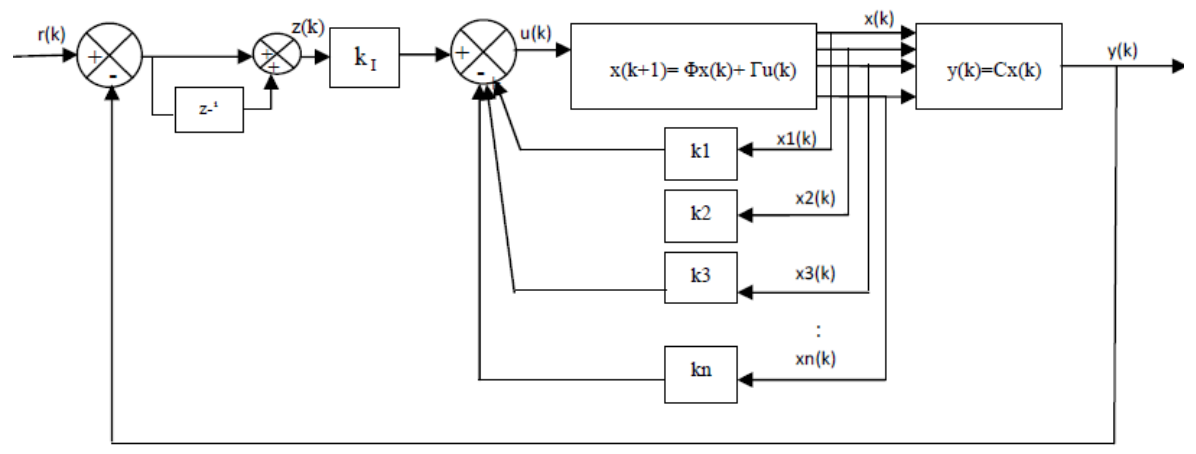

FIG. 2. SERVO SYSTEM STRUCTURE

From Fig. 2, the enhanced state-space model was obtained as described in the next equation, for more details see [10]:

$$
\begin{aligned}
& \mathrm{x}_{\mathrm{d}}(\mathrm{k}+1)=\phi_{\text {aug }} \mathrm{x}_{\mathrm{d}}(\mathrm{k})+\Gamma_{\text {aug }} \mathrm{u}_{\mathrm{d}}(\mathrm{k}), \quad \mathrm{k}=0,1,2, \ldots \\
& \mathrm{y}_{\mathrm{d}}(\mathrm{k})=G_{\text {aug }} \mathrm{x}_{\mathrm{d}}(\mathrm{k})
\end{aligned}
$$

Define the enhanced state as follows:

$$
\phi_{\text {aug }}=\left[\begin{array}{cc}
\phi & 0 \\
-G \phi & 1
\end{array}\right], \Gamma_{\text {aug }}\left[\begin{array}{c}
\Gamma \\
-G \Gamma
\end{array}\right]
$$

And

$$
\begin{gathered}
K^{*}=\left[\begin{array}{ll}
K & -K_{I}
\end{array}\right], \\
\xi(k)=\left[\begin{array}{c}
x_{e}(k) \\
z_{e}(k)
\end{array}\right]=\left[\begin{array}{lll}
x_{e 1} & x_{e 2} & x_{e 3}
\end{array}\right]^{T}, \\
u^{*}(k)=-K^{*} \xi(k)
\end{gathered}
$$

Where $u^{*}(k)$ is the optimal control low and $K$ is the control gain.

\section{B. Optimal LQ-servo Controller}

The optimal LQ-servo problem is to determine the $K^{*}$ matrix of $u^{*}(k)$ to minimize the cost function:

$$
J=\sum_{0}^{\infty}\left(\xi^{T}(k) Q x(k)+u^{* T}(k) R u^{*}(k)\right)
$$

Where,

$k=\left(R+\Gamma^{T} P \Gamma\right)^{-1} \Gamma^{T} P \phi$ and $P=P^{T}$ is the solution matrix of the discrete algebraic Riccatti's equation:

$$
\phi^{T} P \phi-P+Q-\phi^{T} P \Gamma S^{-1} \Gamma^{T} P \phi=0
$$




\section{PARTICLE SWARM OPTIMIZATION OF LQ CONTROLLER}

To overcome the obstacle of selecting the appropriate controller parameters $\left(K^{*}\right)$, Particle Swarm Optimization was used.

The PSO algorithm was fundamentally applied to find the optimal controller parameters $Q$ and $R$, such that a good output response could be obtained.

Since $Q$ has dimensions of $3 \times 3, Q=Q^{T}$ and $R$ is a scalar value, therefore, seven controller parameters were defined, $q_{11}, q_{12}, q_{13}, q_{22}, q_{23}, q_{33}$ and $R$; so, there are seven members in an individual. As a result, the dimension of a population is $n \times 7$ for $n$ individuals in a population.

The optimization method is driven by using the fitness function $\mathrm{F}$, defined bellow:

$$
\text { ITAE }=\sum_{0}^{T} T\left|q_{\text {ref }}-q\right|
$$

$$
\text { Fitness }=1 /(1+\text { ITAE })
$$

The searching proceedings of the proposed LQ-servo based PSO controller were as follows:

Step 1: The seven parameters of the controller were assigned and the population individuals are randomly initialized such as searching points, best positiot, velocities and gbest, position.

Step 2: For each individual, the fitness value $(F)$ was calculated.

Step 3: Each individual's ( $F$ ) was compared with its best position. The best value among the best position was assigned as gbest (best controller parameter).

Step 4: Position and velosity of each individual were modified.

Step 5: If the iterations number is less than the maximum, then go to Step 2. else, go to 6 .

Step 6: The individual with the latest gbest value is assigned as the best controller parameter.

\section{SIMULATION}

In this section, the performance of the designed LQ controller is verified by MATLAB.

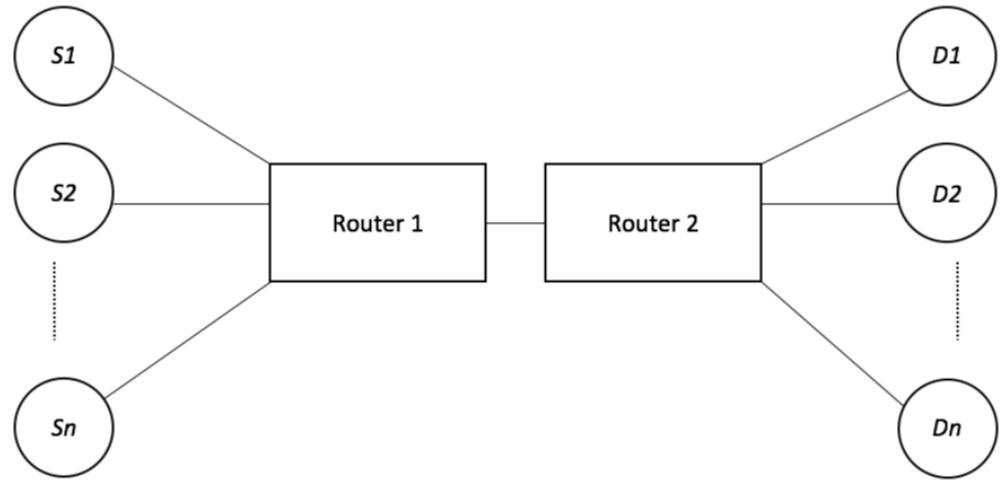

Fig 3. Simulation TOPOLOGY[11]

The designed controller is compared with the PI controller. The TCP/AQM network topology that was used in the simulation is illustrated in Fig.3 with 60 TCP connections, bottleneck link bandwidth is $15 \mathrm{Mbps}$, delay $=20 \mathrm{~ms}$ and the packet size $=500$ bytes. The target queue size changes every 50 seconds with an initial value of 300 , then it drops to 200 then changes to 400 and 200 . The input matrices of the continuous system model are:

$$
A=\left[\begin{array}{cc}
-0.5388 & 0 \\
242.9024 & -4.0650
\end{array}\right], B=\left[\begin{array}{c}
-480.4688 \\
0
\end{array}\right]
$$




$$
C=\left[\begin{array}{ll}
0 & 1
\end{array}\right]
$$

Firstly, the PI controller is examined for comparison reasons. Then, the designed controller (LQ-servo) performance is determined by adding the servo structure and the enhanced state space model is:

$$
\begin{aligned}
& \phi_{\text {aug }}=\left[\begin{array}{ccc}
0.96 & 0 & 0 \\
12.77 & 0.78 & 0 \\
-12.77 & -0.78 & 1
\end{array}\right] \Gamma=\left[\begin{array}{c}
-28.3 \\
-192.7 \\
192.7
\end{array}\right] \\
& G_{\text {aug }}=\left[\begin{array}{lll}
0 & 1 & 0
\end{array}\right]
\end{aligned}
$$

By the proposed PSO method, the optimal value of matrices $Q$ and $\mathrm{R}$, and the control gains were found below:

$$
\begin{aligned}
Q & =\left[\begin{array}{ccc}
0.1000 & 0.6555 & 0.3338 \\
0.6555 & 0.3333 & 0.0 .1211 \\
0.3338 & 0.1211 & 0,8365
\end{array}\right], R=[2] \\
K^{\bullet} & =\left[\begin{array}{lll}
-0.0615 & -0.0281 & -0.0130
\end{array}\right]
\end{aligned}
$$

With the following PSO parameters: population size $=90$, weight factor $w=0.8$, acceleration constants $c_{1}=1.2$ and $c_{2}=0.12$. Fig. 4 shows the system response of the LQ servo based on PSO compared with LQ-servo and PI controllers.

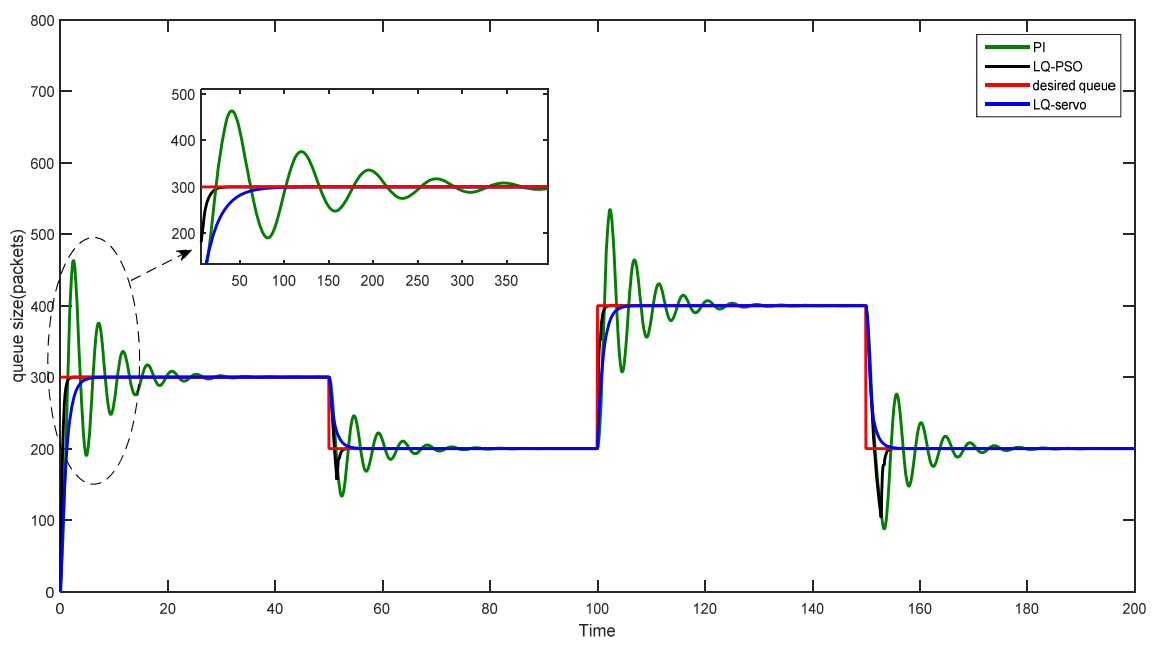

FIG. 4. SYSTEM RESPONSE WITH LQ-SERVO BASED PSO

It is seen that the LQ controller enhances the system response, and as a result, it provides better congestion avoidance compared with the PI controller and the LQ_servo based PSO provides even better congestion avoidance ability than the LQ_servo controller, as shown in table (1).

Rise time, overshoot and settling time were recorded for performance comparison, as illustrated in table (1).

Finally, the performance of the LQ-servo controller was analyzed under various network environments such as varying number of TCP connections $(\mathrm{N})$, which were changed to 300 . The system response is shown in Fig.5, and the results show that the LQ-servo based on PSO controller has the ability to bring the queue size to the desired level. 


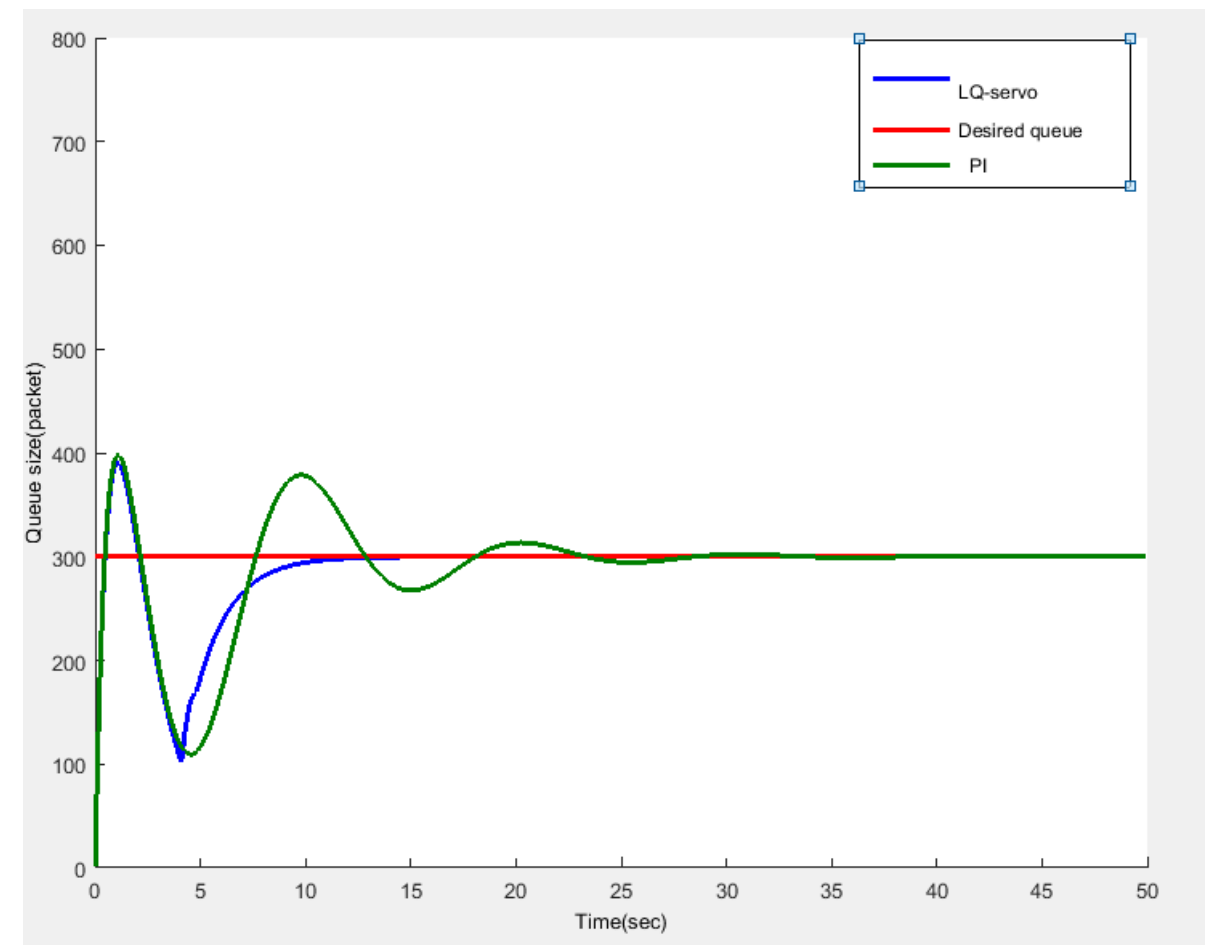

FIG. 5. THE PERFORMANCE OF PI \& LQ-SERVO BASED ON PSO CONTROLLERS WITH N=300

TABLE 1 . SYSTEM RESPONSE FOR TCP WITH DIFFERENT CONTROLLERS

\begin{tabular}{ccccc}
\hline Controller & $\begin{array}{c}\text { Rise Time } \\
\text { (second) }\end{array}$ & Overshoot & $\begin{array}{c}\text { Peak Time } \\
\text { (second) }\end{array}$ & $\begin{array}{c}\text { Settling Time } \\
\text { (second) }\end{array}$ \\
\hline PI & 3.3 & $33 \%$ & 8 & 36 \\
LQ-SERVO & 2.4 & - & - & 8 \\
PSO/LQ-SERVO & 0.83 & - & - & 4 \\
\hline
\end{tabular}

\section{CONCLUSION}

Congestion is a very significant problem in internet networks. In this paper, an AQM model was proposed to solve the congestion problem. The parameters of this AQM based LQ-servo controller were obtained by the PSO algorithm. Then, the PSO-LQ was implemented in MATLAB. The results proved the effectiveness of the proposed controller and showed that it is more effective than the PI in reaching zero steady-state error with better congestion avoidance under the dynamic network environment. Moreover, the proposed controller achieved a smaller delay and faster settling time.

\section{REFERENCES}

[1] V. Jacobson, "Congestion avoidance and control," in ACM SIGCOMM computer communication review, 1988, pp. 314-329.

[2] B. Braden, D. Clark, J. Crowcroft, B. Davie, S. Deering, D. Estrin, et al., "Recommendations on queue management and congestion avoidance in the Internet," 2070-1721, 1998.

[3] S. Floyd and V. Jacobson, "Random early detection gateways for congestion avoidance," IEEE/ACM Transactions on networking, vol. 1, pp. 397-413, 1993.

[4] V. Misra, W.-B. Gong, and D. Towsley, "Fluid-based analysis of a network of AQM routers supporting TCP flows with an application to RED," in ACM SIGCOMM Computer Communication Review, 2000, pp. 151-160.

[5] C. V. Hollot, V. Misra, D. Towsley, and W.-B. Gong, "On designing improved controllers for AQM routers supporting TCP flows," in Proceedings IEEE INFOCOM 2001. Conference on Computer 
Communications. Twentieth Annual Joint Conference of the IEEE Computer and Communications Society, 2001, pp. 1726-1734.

[6] C. Hollot, V. Misra, D. Towsley, and W.-B. Gong, "A control theoretic analysis of RED," in INFOCOM 2001. Twentieth annual joint conference of the IEEE computer and communications societies. Proceedings. IEEE, 2001, pp. 1510-1519.

[7] S. Floyd and V. Jacobson, "On traffic phase effects in packet-switched gateways," Internetworking: Research and Experience, vol. 3, pp. 115-156, 1992.

[8] M. Zhenwei, Q. Junlong, and Z. Lijun, "Design and Implementation: Adaptive Active Queue Management Algorithm Based on Neural Network," in Computational Intelligence and Security (CIS), 2014 Tenth International Conference on, 2014, pp. 104-108.

[9] K. Ogata, "Modern control engineering,(1997)," ISBN: 0-13-227307-1, pp. 299-231, 2013.

[10] M. Z. Al-Faiz and S. S. Sabry, "Optimal linear quadratic controller based on genetic algorithm for TCP/AQM router," in Future Communication Networks (ICFCN), 2012 International Conference on, 2012, pp. 78-83.

[11] B. A. Sadek, T. El Houssaine, and C. Noreddine, "A robust PID controller for active queue management framework in congested routers," in 2017 Intelligent Systems and Computer Vision (ISCV), 2017, pp. 1-6. 